\title{
A Rare Case of Cholestasis: Arthrogryposis, Renal Tubular Disorder and Cholestasis Syndrome
}

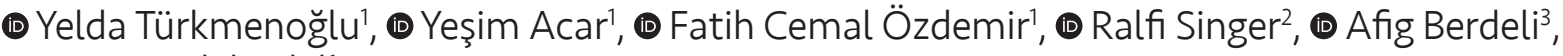 \\ (1) Servet Erdal Adal ${ }^{1}$
}

1'stanbul Okmeydanı Training and Research Hospital, Clinic of Pediatric, İstanbul, Turkey

2istanbul Okmeydanı Training and Research Hospital, Clinic of Dermatology, İstanbul, Turkey

${ }^{3}$ Ege University Faculty of Medicine, Pediatric Molecular Research Laboratory, İzmir, Turkey

\begin{abstract}
Arthrogryposis, renal tubular dysfunction and cholestasis (ARC) syndrome is a rare, autosomal recessive multisystem disorder. Severe growth retardation, ichthyosis, recurrent febrile disease, platelet abnormalities, sensorineural hearing loss, hypotonia and corpus callosum dysgenesis were later included as further features of this syndrome. We present a case of ARC syndrome diagnosed by genetic analysis.

Keywords: Arthrogryposis, ichthyosis, cholestasis, renal tubular disorder
\end{abstract}

\section{Introduction}

Arthrogryposis, renal tubular dysfunction, cholestasis (ARC) syndrome was originally described in 1973 by LutzRichner and Landolt (1) severe growth retardation, ichthyosis, recurrent febrile disease, platelet abnormalities, sensorineural hearing loss, hypotonia and corpus callosum dysgenesis were later included as further features of the syndrome $(1,2)$. This multisystemic disease due to an autosomal recessive hereditary transmission occurs as a result of VPS33B or VIPAR gene mutations $(3,4)$. Most of the patients are lost during the first year of their lives due to dehydration, acidosis, sepsis and particularly profuse bleeding due to diagnostic invasive interventions $(1,2,4)$. We present here a case of ARC syndrome diagnosed by genetic analysis.

\section{Case Report}

A thirty-five-day old female infant was referred to our hospital with jaundice and lack of weight gain. The parents of the infant, born at term weighing $2.620 \mathrm{~g}$, were second degree relatives. At the referral time, her weight was $2.800 \mathrm{~g}$ (<3 p\%), height $51 \mathrm{~cm}(3-10 \mathrm{p} \%)$ and head circumference 33 $\mathrm{cm}(<3 \mathrm{p} \%)$. Physical examination revealed jaundice of the skin and sclera, generalized hypotonia, dry skin, bilateral club feet and hepatomegaly. There was no history of acholic stool and it was not observed during follow up either. Laboratory results were as follows: white blood cell count 15.240/ $\mathrm{mm}^{3}$, hemoglobin $7.9 \mathrm{~g} / \mathrm{dL}$, platelet count $397.800 / \mathrm{mm}^{3}$, C-reactive protein $1.45 \mathrm{mg} / \mathrm{L}$, glucose $77 \mathrm{mg} / \mathrm{dL}$, aspartate aminotransferase (AST) $133 \mathrm{U} / \mathrm{L}$, alanine aminotransferase

\section{Address for Correspondence}

Yelda Türkmenoğlu MD, istanbul Okmeydanı Training and Research Hospital, Clinic of Pediatric, Istanbul, Turkey Phone: +905324370145 E-mail:yldtrkmngl@hotmail.com ORCID ID: orcid.org/0000-0001-7472-8748 Received: 25.04.2017 Accepted: 05.06.2017

${ }^{\circ}$ Copyright 2018 by Ege University Faculty of Medicine, Department of Pediatrics and Ege Children's Foundation The Journal of Pediatric Research, published by Galenos Publishing House. 
(ALT) $169 \mathrm{U} / \mathrm{L}$, total bilirubin $9.72 \mathrm{mg} / \mathrm{dL}$, direct bilirubin $4.51 \mathrm{mg} / \mathrm{dL}$, alkaline phosphatase (ALP) $1142 \mathrm{U} / \mathrm{L}$, gamma glutamyl transpeptidase (GGT) $31 \mathrm{U} / \mathrm{L}$, prothrombin time 11.81 secs, international normalized ratio 1.03 and activated partial thromboplastin time 20.39 secs. Peripheral smear findings showed leucocytosis and normal thrombocytes. Thyroid function tests, viral hepatitis markers, alfa fetoprotein levels were normal. Tandem mass spectrometry and urinary organic acid analysis were normal but urine amino acid chromatography showed generalized aminoaciduria. Blood $\mathrm{pH}$ was 7.21, bicarbonate $17 \mathrm{mmol} / \mathrm{L}$ and base deficit -8.3. urinary $\mathrm{pH}$ was 8.5 with glucosuria and proteinuria and fractional phosphate absorption was 0.4 . These findings led to the diagnosis of proximal renal tubular acidosis (RTA). The liver and biliary system were normal in abdominal ultrasonography (USG), but pelvic ectasia and dysplasia were found at the left kidney. Cranial USG and tomography were normal. Hip ultrasound revealed a grade 3 dislocation on the right side and a grade II dislocation on the left. Bilateral sensorineural hearing loss was observed under brainsteam evoked response audiometry testing. The dermatological examination was consistent with ichthyosis. Based on these clinical and laboratory findings, ARC syndrome was considered. Due to expected severe bleeding disorder and lack of family consent, organ biopsies were not performed and genetic analysis revealed a homozygotic p.Cly514 Ser mutation in the VPS33B gene (Figure 1).

During follow up, growth retardation and ichthyosis progressively increased, growth parameters were below the third percentile at the age of three years. Cranial magnetic resonance imaging (MRI) showed corpus callosum dysgenesis and cerebellar displacement called Arnold Chiari malformation Type I. There were several oral bleeding episodes during teething. These bleedings could be stopped

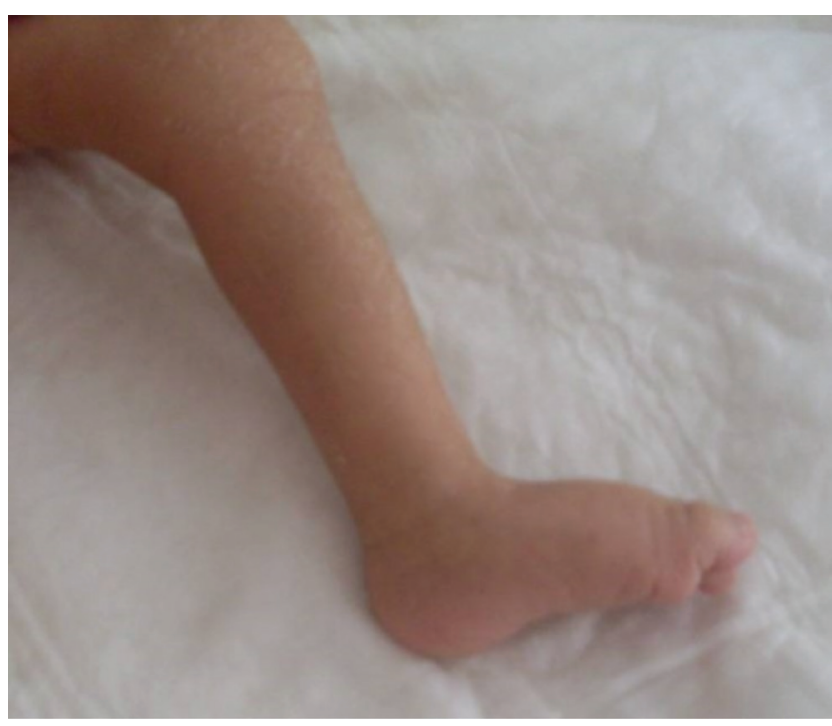

Figure 1. Club foot, icterus and dry-scaly skin of patient only by applying local anticoagulant. The patient had multiple febrile episodes and recurrent suppurative otitis media with Pseudomonas aeruginosa growth in ear culture due to hearing aid apparatus. The patient was lost in her last febrile episode because of severe intractable septicaemia.

\section{Discussion}

ARC syndrome is a rare disease and its incidence is not known. Most cases of ARC syndrome have been reported in Pakistani, Saudi Arabia, Oman, North Africa, Asia, Italy and Portugal. In Turkey, the disease was described for the first time in 2005 in two siblings and only a few cases have been added to them since then $(5,6)$. However, in a study including a series of 90 infants with cholestasis in Korea, $46 \%$ were diagnosed as extrahepatic biliary atresia, 30\% as neonatal hepatitis and $7 \%$ were diagnosed as having ARC syndrome due to a demonstrated VPS33B mutation (7). It was believed that some patients die without being diagnosed properly.

Arthrogryposis, which is thought to occur as a result of the degeneration of frontal motor neurons, is one of the distinguishing features of this syndrome. Characteristics of arthrogryposis include contractures, clubfeet, fractures and hip dislocations $(4,6)$. Hip dislocation and clubfeet in our patient were considered as characteristics of arthrogryposis. Renal tubular dysfunction is observed in all patients and it is usually Fanconi type RTA associated with glucosuria, phosphaturia and proteinuria and less frequently as renal diabetes insipidus. Renal USG reveals nephrocalcinosis and dysplastic kidneys and renal biopsy shows glomerulosclerosis, tubular degeneration, calcification and glomerulocystic appearance $(4,6,7)$. In our patient, there were dysplastic kidneys and Fanconi type RTA. Cholestasis and hepatomegaly exist in all patients, a mild elevation of ALT, AST and ALP levels in association with a normal GGT level are particularly seen in this syndrome (1-4). Normal GGT levels are also seen in progressive familial intrahepatic cholestasis syndromes but without arthrogryposis and ichthyosis. Liver histology is described as a decrease in biliary ductus and proliferation, giant cell formation, pigment deposition, extramedullary hematopoesis and portal fibrosis which are non-specific features $(4,7)$. Recently, because of a high risk of profuse bleeding, genetic analysis is recommended instead of biopsy (4). In our patient, ALT, AST and ALP levels were elevated and GGT was normal. A liver biopsy was not performed because of severe bleeding risk and family consent was lacking; thus, the diagnosis was confirmed by genetic analysis. Severe growth retardation is seen in all patients and severe acidosis, diarrhea, recurrent febrile diseases and renal losses account for this growth retardation $(2,4,7)$. Our patient also had severe growth retardation despite all the supportive therapies. Ichthyosis may not be present at birth in many cases and it usually occurs after the first month of life 
(4). Our patient had ichthyosis unresponsive to treatment, worsening gradually over time. The central nervous system symptoms of this syndrome are hypotonia, sensorineural hearing loss and corpus callosum dysgenesis or agenesis $(1,4,7)$. Our patient was using a hearing aid apparatus due to sensorineural hearing loss and cranial MRI had shown corpus callosum dysgenesis and Arnold Chiari Type I malformation. It is not known if Arnold Chiari malformation is a component of ARC syndrome or a coincidence because there was no published data to be found in the medical literature. Tendency to spontaneous bleeding is reported in these patients and it is considered that Grey Platelet syndrome and a dysfunction of alpha granules are the causes of these bleeding episodes $(4,8)$. In a case report, spontaneous nasal bleeding could be stopped only by the delivery of a platelet suspension (8). Our patient had several prolonged oral mucosal bleeding episodes despite normal coagulation tests. These bleedings were stopped by use of local anticoagulants and platelet suspensions were not given. Genetic mapping of ARC syndrome was described as a mutation of chromosome $15 q 26.1$ in VPS33B gene. This gene encodes the VPS33B protein that is involved in the vesicular trafficking pathway (4). This protein, existing in various organs, is involved in intercellular synaptic transmission, vesicular exocytosis and general secretion. Akbar et al. (9) have shown that the VPS33B gene is involved in phagosome and endosome maturation and is responsible for the recognition of microorganisms. Staphylococcus species, Escherichia coli and Pseudomonas aeruginosa were shown as causative agents $(8,9)$. Episodes of suppurative otitis media caused by Pseudomonas aeruginosa were observed in our patient. Treatment in ARC is usually symptomatic. However, recently, there has been a reported case of a child with ARC syndrome from Iran undergoing a liver transplantation and still surviving after five years (10). Further prognostic improvements are expected for this condition. In conclusion, ARC syndrome is a cause of cholestasis and it should be particularly kept in mind in certain geographical locations including Turkey. In an infant presenting with cholestasis that is associated with ichthyosis and renal tubular dysfunction, ARC syndrome should be considered.

\section{Ethics}

Informed Consent: The verbal consent was taken from the patient's parents.
Peer-review: Externally peer-reviewed.

\section{Authorship Contributions}

Surgical and Medical Practices: Y.T., A.B., F.C.Ö., Concept: Y.T., Y.A., Design: Y.T., S.E.A., Data Collection and Processing: Y.T., A.B., R.S., Analysis and Interpretation: Y.T., S.E.A., Literature Search: Y.T., R.S., F.C.Ö., Writing: Y.T., Y.A., R.S., S.E.A.

Conflict of Interest: No conflict of interest was declared by the authors.

Financial Disclosure: The authors declared that this study received no financial support.

\section{References}

1. Lutz-Richner AR, Landolt RF. Familiare Gallengansmissbildungen mit tubularer Neireninsurfizienz. Helv Paediatr Acta 1973;28:112.

2. Nili F, Akbari-Asbaghe P, Oloomi-Yazdi Z, et al. Wide spectrum of clinical features in a case of arthrogryposis-renal tubular dysfunction-cholestasis syndrome. Arch Iran Med 2008;11:56972.

3. Gissen P, Johnson CA, Morgan NV, et al. Mutations in VPS33B, encoding a regulator of SNARE-dependent membrane fusion, cause arthrogryposis-renal dysfunction-cholestasis (ARC) syndrome. Nat Genet 2004;36:400-4.

4. Zhou Y, Zhang ). Arthrogryposis-renal dysfunction-cholestasis (ARC) syndrome: from molecular genetics to clinical features. Ital J Pediatr 2014;40:77.

5. Tekin N, Durmuş-Aydoğdu S, Dinleyici EC, Bör O, Bildirici K, Akşit A. Clinical and pathological aspects of ARC (arthrogryposis, renal dysfunction and cholestasis) syndrome in two siblings. Turk J Pediatr 2005;47:67-70.

6. Arhan E, Yusufoğlu AM, Sayli TR. Arc syndrome without arthrogryposis, with hip dislocation and renal glomerulocystic appearance: a case report. Eur J Pediatr 2009;168:995-8.

7. Jang JY, Kim KM, Kim $\mathrm{GH}$, et al Clinical characteristics and VPS33B mutations in patients with ARC syndrome. J Pediatr Gastroenterol Nutr 2009;48:348-54.

8. Saadah OI, Bokhari BE, Alshaeri TM, Jastaniah W. Haematological manifestations of arthrogryposis-renal dysfunction-cholestasis (ARC) syndrome: a case report. Arab J Gastroenterol 2013;14:268.

9. Akbar MA, Mandraju R, Tracy C, Hu W, Pasare C, Krämer H. ARC Syndrome-Linked Vps33B Protein Is Required for Inflammatory Endosomal Maturation and Signal Termination. Immunity 2016;45:267-79.

10. Dehghani SM, Bahador A, Nikeghbalian S, et al. Liver transplant in a case of arthrogryposis-renal tubular dysfunction-cholestasis syndrome with severe intractable pruritus. Exp Clin Transplant 2013;11:290-2. 